\title{
Fitzpatrick Skin Type II
}

National Cancer Institute

\section{Source}

National Cancer Institute. Fitzpatrick Skin Type II. NCI Thesaurus. Code C74570.

Skin which is white and it usually burns easily when exposed to the sun. It is usually seen in people with blond or red hair and blue or hazel eyes. 